\title{
Social Motivational Processing and Interpersonal Function in Aging Cocaine Smokers
}

Bedi $\mathrm{G}^{1,2^{*}}$, Hao $\mathrm{X}^{1}$, Van Dam $\mathrm{NT}^{3}$, Cooper $\mathrm{ZD}^{1}$, Rubin $\mathrm{E}^{1}$, Vadhan $\mathrm{NP}^{4}$, Marino $\mathrm{L}^{1}$, Haney $\mathrm{M}^{1}$

${ }^{1}$ Department of Psychiatry, Columbia University Medical Center and New York State Psychiatric Institute, NY, USA

${ }^{2}$ Centre for Youth Mental Health, University of Melbourne, and Orygen National Centre of Excellence in Youth Mental Health, Melbourne, Australia

${ }^{3}$ School of Psychological Sciences, University of Melbourne. Melbourne, Australia ${ }^{4}$ Hofstra-Northwell School of Medicine and Feinstein Institute for Medical Research, NY, USA

Abstract: 250

Main Text: 4849

Figures: 5

Tables: 2

Supplemental material: Two tables

This is the author manuscript accepted for publication and has undergone full peer review but has not been through the copyediting, typesetting, pagination and proofreading process, which may lead to differences between this version and the Version of Record. Please cite this article as doi: 10.1111/adb.12669

This article is protected by copyright. All rights reserved. 
* Address for correspondence: Gillinder Bedi DPsych, Orygen National Centre of Excellence in Youth Mental Health. 35 Poplar Road, Parkville, Victoria, Australia, 3052. Phone: +61 3 9342 3734. Email: gill.bedi@unimelb.edu.au.

This article is protected by copyright. All rights reserved. 


\section{ABSTRACT}

Illicit drug use among aging cohorts is increasing, yet little is known about functional impairments in older drug users. Given the importance of social integration for aging, and documented social decrements in cocaine users, we examined social function and its neurocognitive substrates in aging cocaine users relative to carefully matched non-cocaine users. Regular ( $\geq$ twice/week), long-term ( $\geq 15$ years) cocaine smokers 50-60 years old (COC; $n=22 ; 4$ females) and controls (CTRL; $n=19 ; 4$ females) underwent standardized probes of social reward and threat processing during functional Magnetic Resonance Imaging (fMRI) and a behavioral facial affect recognition task. Self- and peer-reports of daily interpersonal function were also collected. COCs, and CTRLs reporting current marijuana or alcohol use, were tested after 4 drug-free inpatient days. COCs had pronounced problems in daily social function relative to CTRLs indicated by both their own and their peers' reports. Compared to CTRLs, COCs had stronger amygdala responses to social threat versus control stimuli, with no other differences in social processing or cognition. Aging cocaine users appear to have marked, generalized difficulties in 'real-world' interpersonal function but largely intact social processing on laboratory-based measures when compared to appropriately matched controls and tested under well-controlled conditions. Daily social difficulties may be related to transient factors such as acute/residual drug effects or cocainerelated changes in health behaviors (e.g. disrupted sleep, poor diet). These data suggest that interpersonal function may be a valid intervention target for aging cocaine users, and warrants further study in older drug users. 
Keywords: cocaine, aging, social cognition, social reward, social threat, interpersonal problems

This article is protected by copyright. All rights reserved. 


\section{INTRODUCTION}

Until recently, aging has not been a focus of addiction research, with older drug users historically underrepresented (Rosen et al., 2013). However, people over 50 now represent $21 \%$ of past-month illicit drug users in the US (SAMSHA, 2016). This number will likely increase with continued aging of the 'baby boomers', a large cohort with greater illicit drug use than previous generations (Han et al., 2009; Rao and Roche, 2017). Thus, older substance users will likely present challenges to public health systems in coming years (Rao and Roche, 2017), particularly given potential interactions between drug effects and aging (Dowling et al., 2008).

Cocaine smokers are one such aging population (Kalapatapu et al., 2011; Yarnell, 2015). In 2014, an estimated 372,000 people in the US over 50 had used cocaine or "crack" in the past month, a number that increased to 562,000 in 2015 (SAMSHA, 2016). These data are supported by case reports, with older cocaine users presenting in drug treatment (Kouimtsidis and Padhi, 2007), emergency (Yarnell, 2015), and urgent care (Yarnell, 2015) settings. Presentation in generalist medical settings underlines the greater likelihood of adverse health implications of cocaine use in aging relative to younger users. Indeed, one might expect adverse effects in this population to cause greater disability and potentially be more difficult to treat, highlighting the need to identify additional ways to facilitate functional recovery in this group.

Assessing social function and its neurobiology is particularly important in aging drug users because social integration is a key determinant of successful aging (Fuller-Iglesias and 
Rajbhandari, 2016). Even in younger groups, cocaine use is associated with marked social difficulties: relative to non-users, users are more likely to experience and perpetrate violence, including homicide (Kuhns et al., 2009), sexual assault (Afful et al., 2010), and domestic violence (Moore et al., 2008). They have high rates of unemployment (Gfroerer and Brodsky, 1993), homelessness (Eyrich-Garg et al., 2008), and loss of child custody (Eiden et al., 2007). Moreover, cocaine users report that they experience a range of social adjustment problems (Cunha et al., 2011). Although some problems likely relate to cocaine's illegality, recent evidence suggests that cocaine users have disruptions in key domains of social cognition and information processing, potentially contributing to dysregulated interpersonal behavior. Relative to non-users, cocaine users exhibit decreased facial emotion recognition, particularly for threat-related emotions (Ersche et al., 2015; Kemmis et al., 2007; Morgan and Marshall, 2013). Another study found decreased emotion recognition in cocaine users due to lowered identification of emotion from vocal prosody and difficulty integrating cross-modal (prosody/facial) affective information. Emotion recognition difficulties also predicted a smaller social network, suggesting that these difficulties may have significant consequences (Hulka et al., 2013).

Whereas most research with cocaine users has focused on social cognition (Ersche et al., 2015; Kemmis et al., 2007; Morgan and Marshall, 2013), two recent studies examined social reward processing, a key dimension of social motivation. In one, cocaine users showed blunted social reward during virtual interaction relative to controls, indicated by self-report and physiological measures. These differences were associated with decreased activation during 
simulated interaction in orbitofrontal cortex, a region involved in valuation and reward processing (Preller et al., 2014). A subsequent study reported reduced ventromedial prefrontal cortex (VmPFC) signaling during both social and non-social reward receipt in cocaine users compared to controls, suggesting generalized blunting of non-drug reward processing (Tobler et al., 2016).

Findings of deficient social reward processing are consistent with valuation theories of addiction, which propose that Substance Use Disorders (SUDs) are perpetuated by overvaluation of drugs coupled with undervaluation of non-drug reinforcers (Volkow et al., 2011). Moreover, disrupted social cognition and motivation have potentially profound implications: they may hinder engagement in psychotherapy, the gold standard treatment for cocaine users in the absence of approved medications. They may also limit access to a key non-drug reinforcer by disrupting meaningful social bonds. Given the known importance of social integration in aging, these effects could be particularly damaging in older users. To date, no studies have assessed social function in aging cocaine users.

Here, we assessed social processing in aging cocaine smokers relative to demographically-matched controls. We examined (1) social cognition (facial emotion recognition; FER); (2) social reward processing; (3) social threat processing; and (4) daily interpersonal function (assessed by self- and peer-report). We endeavored to control for several confounds known to occur in cross-sectional studies, including acute and residual drug effects (Kuypers et al., 2015). Relative to controls, we expected aging cocaine users with short-term abstinence to show blunted identification of threatening facial emotions, 
accompanied by dampened social threat processing. We further expected that they would have blunted social reward processing and difficulties in day-to-day social function.

\section{MATERIALS AND METHODS}

\section{Participants}

Cocaine Smokers (COC): Healthy, non-treatment-seeking cocaine smokers verbally fluent in English were recruited. Participants were 50-60 year-old right-handed male and nonpregnant females reportedly smoking cocaine $\geq 2$ times/week for the past six months (supported by urine toxicology). COC were required to report cocaine use initiation $\geq 20$ years prior, with $\geq$ weekly use for $\geq 15$ years. COC-specific exclusion criteria were: current Substance Dependence except cocaine or nicotine [all diagnoses according to DSM-IV criteria (APA, 1994)]; past Substance Dependence except cocaine, marijuana, alcohol, or nicotine; and current Substance Abuse except cocaine, alcohol, marijuana, or nicotine.

Controls (CTRL): Controls were 50-60 year-old right-handed male and females fluent in English. They were excluded for: current Substance Dependence except nicotine; past Substance Dependence except marijuana, alcohol, or nicotine; and current Substance Abuse except marijuana, alcohol, or nicotine. We aimed to recruit CTRLs with marijuana, alcohol and/or nicotine because cocaine users also frequently use these (Vadhan et al., 2014). We included controls reporting up to 10 lifetime uses of cocaine with no use in the past year, because many recruited controls reported remote cocaine experimentation, consistent with high availability of cocaine among urban populations in the 1980s (Cornish and O'Brien, 1996).

This article is protected by copyright. All rights reserved. 
COCs and CTRLs were excluded for: past year mood, anxiety, or eating disorder; lifetime mania or psychosis; reported history of neurological illness or autism; medical conditions contraindicating participation or likely to affect social cognition; and MRI contraindication.

Participants provided written informed consent as approved by the New York State Psychiatric Institute (NYSPI) Institutional Review Board.

\section{Experimental Protocol}

Participants underwent comprehensive screening including blood and urine analysis, ECG, physical examination, and psychiatric assessment [Structured Clinical Examination for DSM-IV Diagnoses; SCID (First et al., 2002)]. A doctoral-level researcher collected drug use history. Participants attended up to five screening sessions, with urine toxicology (5-Panel drug screen, All Test North America, Gilbert, AZ), and breathalyzer (Select S80, BACTrack, San Francisco, CA) samples tested in each session, minimizing the potential for misrepresentation of drug use. Female participants were tested for pregnancy (Alere hCG Dipstick, Alere, Orlando, FL).

Following screening, participants were admitted into the study. COC participants and CTRLs with current cannabis or regular alcohol use completed the protocol as inpatients to control for acute and residual drug effects, as well as to ensure adequate sleep and food intake before testing. They were admitted to the NYSPI Clinical Research Unit for 5 days, with data collection on the final inpatient day. On the morning of testing, following breakfast, participants were tested for alcohol, illicit drugs, and pregnancy (females) and were trained in 
the tasks. They completed a self-report measure of mood. They then underwent a 90-minute MRI scan session including social and non-social reward and threat tasks (below). Following the scan, participants were provided with lunch prior to undergoing a cognitive battery, including a facial emotion recognition task and non-social cognitive measures. Data from the non-social cognitive tests address different hypotheses and will be reported separately. Inpatient participants were compensated, debriefed and discharged the morning after data collection.

CTRL participants who reported no current marijuana or regular alcohol use (biochemically verified during screening) completed the testing during a single outpatient day. They were asked to abstain from alcohol for 4 days, from caffeine and any medications for 24 hours, and from cigarettes for 12 hours prior to the session. The outpatient session followed the same timeline as inpatient testing. Upon arrival, participants were tested for alcohol, illicit drugs, and pregnancy (females). They were provided with breakfast and trained in the MRI tasks. They were scanned and then provided with lunch. In the afternoon they underwent cognitive testing. At session completion, outpatient participants were compensated, debriefed, and discharged.

Cigarette smokers were allowed to smoke a single cigarette approximately 1 hour before the MRI to reduce effects of nicotine withdrawal.

\section{Measures}

$\underline{\text { Social Cognition }}$ 
Facial Emotion Recognition (FER): FER was assessed with a modification of an existing task (Bedi et al., 2016), assessing accuracy identifying happy, sad, fearful, angry, disgusted, and surprised expressions, morphed from neutral to the prototype emotion (100\%) in $10 \%$ increments. Faces $(N=250)$ were presented in randomized order for $500 \mathrm{~ms}$, followed by a prompt to choose the emotion. Stimuli ( $50 \%$ female; $50 \%$ male) were taken from the NIMSTIM (Tottenham et al., 2009), morphed using FantaMorph (Abrosoft, Los Angeles, CA). Stimuli were matched to expected sample race/ethnicity [primarily Black or Hispanic (Bedi et al., 2016)]. The main outcomes were proportion correct and the number of false positives by emotion. Reaction time (RT) for correct identifications was also analyzed.

Motivational Processing

Social Reward Processing - Social Incentive Delay (SID): Behavioral and neural social reward processing was measured with a variant of the Monetary Incentive Delay Task (MID), the Social Incentive Delay [SID (Spreckelmeyer et al., 2009)]. The SID follows the MID trial structure (Figure 1). An initial cue indicating the level of reward for the upcoming trial (no, low, or high reward) is followed by a variable anticipation period ending with a simple reaction time task. Successful trials result in feedback: for high reward, participants see an exuberantly smiling face and the words "You did great", whereas for low reward trials, they see a happy closed-mouth smiling face with the words "You did good". Successful no reward trials result in presentation of facial stimuli that have been graphically dysmorphed to remove features, with the words "You did ok". Unsuccessful trials result in presentation of the featureless facial stimuli coupled with either "You did not do great", "You did not do good", or "You did not do 
ok". Stimuli (Tottenham et al., 2009) were 50\% male and 50\% female and matched to expected sample race. Required reaction times were adjusted online to maintain a hit rate approximating 66\%. The SID comprised 72 trials in two runs of 6 mins 12 secs each. The main epoch for fMRI analysis was the anticipation phase (all trials) and the outcome phase (successful trials only).

Non-Social Reward - Monetary Incentive Delay (MID): To assess whether differences in the SID were specific to social motivation, we also included the MID (Knutson et al., 2000). The MID structure was parallel to the SID, with $\$ 0, \$ 1$, and $\$ 2$ corresponding to no, low, and high reward conditions. Participants could earn a maximum of $\$ 66$; monetary earnings were added to study payments. SID and MID runs were interleaved, with order of presentation counterbalanced.

Social and Non-Social Threat Processing - Adapted Emotional Face Processing: We measured social and non-social threat response with a variant of the Emotional Face Processing (EFP) task (Hariri et al., 2002). This involved presentation of 36, 20-sec blocks comprising 4 stimuli across the following conditions: Social Threat (fearful/angry faces); Social Non-Threat (happy/neutral faces); Non-Social Threat (threatening pictures without humans e.g. snakes or spiders); Non-Social Non-Threat (neutral pictures without humans e.g. mushrooms); and Shapes (sensorimotor control). Each screen consisted of a single image at the top and two images below. One of the two bottom images was identical to the top image; participants selected the image on the bottom that was the same as the top image. Facial stimuli were taken from the NIMSTIM (Tottenham et al., 2009), and were 
matched to expected sample racial characteristics. Non-social stimuli were from the International Affective Picture System [IAPS (Lang et al., 1997)]. The EFP comprised 3 runs of 4 min 12 seconds each. The EFP reliably activates bilateral amygdala (Hariri et al., 2002). Daily Interpersonal Function

Inventory of Interpersonal Problems (IIP) Self and Peer Versions: Interpersonal function was measured with the IIP Self and Peer Versions (Horowitz et al., 2000). The IIP consists of 64 items requiring participants/peers to report the extent to which the participant experiences a range of interpersonal problems (e.g. difficulty trusting others, being assertive etc.) on a 5-point scale. The IIP yields an overall index as well as 8 subscale scores: Domineering/Controlling, Vindictive/Self-Centered, Cold/Distant, Socially Inhibited, Nonassertive, Overly Accommodating, Self-Sacrificing, and Intrusive/Needy. The IIP detects interpersonal problems in substance users (Kornreich et al., 2002). For peer reports, participants provided contact details for two friends/relatives whom they had known for $\geq$ six months with whom they had $\geq$ weekly contact. Staff contacted nominated peers, obtained verbal consent, and completed the IIP-Peer over the phone. Peers who completed the IIPPeer were asked to send in a signed consent form; they were subsequently mailed a $\$ 20$ bank check. One IIP-Peer was completed per participant.

Mood and Psychiatric Symptoms

Positive and Negative Affect Schedule (PANAS): Before testing, we used the PANAS (Watson et al., 1988) to assess state affect. The PANAS comprises 10 positive and negative 
affect adjectives; participants rate the extent to which each describes their current affect on a 5-point scale, yielding a Negative and a Positive Affect score.

Beck Depression Inventory (BDI): The BDI (completed during screening) comprises 21 items measuring components of depression, each of which are answered on a 4-point Likert Scale, yielding a single score (Beck and Steer, 1993).

State Trait Inventory of Cognitive and Somatic Anxiety (STICSA): The STICSA (Van Dam et al., 2013), completed at screening, comprises 21 items measured on a 4-point Likert scale yielding a single score.

Trauma Assessment for Adults: Exposure to traumatic events was assessed with the Trauma Assessment for Adults - Brief Revised Version [TAA-BR (Cusack et al., 2004)], which comprises 12 items describing potentially traumatic experiences (e.g. military combat, major accidents). Participants endorse whether they have ever experienced each item (yes/no). The TAA-BR yields a single score.

\section{MRI Acquisition Parameters}

Images were acquired on a 3T GE scanner. During the study, the MRI Research Unit at NYSPI replaced the GE Signa with a GE MR750. Twenty participants (13 COC) were scanned on the old scanner and 20 (8 COC) on the new (one subject did not provide usable fMRI data). There was no between-group difference in proportion of participants scanned on each magnet $\left(X^{2}(1)=2.5, p>.05\right)$, however scanner type was included as a betweensubjects covariate in all MRI analyses. For the SID and MID, blood oxygenation leveldependent (BOLD) images were acquired from 32 axial $3.5 \mathrm{~mm}$ thick slices (0.5 mm gap) 
aligned with the AC-PC line using a T2*-sensitive spiral in and out acquisition sequence (TR $=2000 ; \mathrm{TE}=27$; flip angle $=77 ; 64 \times 64$ matrix, $24 \mathrm{~cm}$ FOV) designed to minimize orbitofrontal signal loss (Glover and Law, 2001). For EFP, BOLD images were acquired from 34 AC-PCaligned 3.5mm thick slices (0.5 mm gap) using EPI acquisition $(T R=2000 ; T E=30$; flip angle $=77 ; 64 \times 64$ matrix, 24cm FOV). Each functional run started with 6 dummy volumes to establish signal equilibrium. We also collected a standard high-resolution T1-weighted anatomical scan.

\section{Statistical Analysis}

Behavioral Data: Demographic and drug-use data were compared between groups using chi-squared tests and independent samples t-tests. IIP data were subject to mixed Analyses of Variance (ANOVAs), with subscale as the within-subject factor and group as the between-subject factor. FER data were analyzed with 2-factor ANOVA (emotion $x$ group). For FER accuracy and misclassifications, only responses with reaction times (RTs) greater than 200ms were included to exclude anticipatory errors (Ratcliff, 1993), leading to removal of less than $1 \%$ of data. For RT, we included only correct items; we also excluded items with RTs less than 200 ms or more than 3.29 standard deviations (SDs) from group means (Ratcliff, 1993) resulting in removal of less than $2 \%$ of data. SID and MID accuracy and RTs (correct responses only) were subject to mixed ANOVAs (level of reward x group). Effects of reward level were assessed with linear contrasts. EFP accuracy was subject to mixed ANOVA, with threat (threat vs. non-threat) and social status (social vs. non social) as within-subjects variables and group as a between-subject variable. Isolated univariate outliers with z-scores 
$>3.29$ were truncated to 1 increment more/less than the second lowest/highest value; in the case that no change in the results was observed, data were retained in original form. Data for SID accuracy are presented after truncation of two outlying data points. Data for EFP accuracy are presented after truncation of 4 outlying data points. Alpha was set at 0.05 for omnibus analyses with a corrected threshold of 0.01 for post-hoc analyses. Analyses were conducted using IBM SPSS Statistics 23 (IBM, Armonk, NY).

fMRI Data: fMRI preprocessing and analyses were carried out using FEAT (FMRI Expert Analysis Tool) version 6.00, FSL (FMRIB Software Library, version 5.0.9, www.fmrib.ox.ac.uk/fsl). Standard preprocessing was applied, including brain extraction (BET), motion correction (MCFLIRT), slice-timing correction, high pass temporal filtering (90 s cutoff), and spatial smoothing (5 mm FWHM Gaussian kernel). Spiral data were corrected for physiological noise using PHYCAA+ (Churchill and Strother, 2013). Functional images were then coregistered to the T1-weighted anatomical image of the same subject, using FLIRT with 12 degree of freedom (DOF) affine transformation, then to the MNI152 atlas using combined affine (12 DOF) and nonlinear registration (FNIRT with a warp resolution of 10 $\mathrm{mm})$. Three-level analysis was performed using a mixed-effects model that treated participants as a random effect. First level analysis was conducted in the native image space. Regressors for blocks (EFP) and events (SID/MID) were convolved with a canonical doublegamma hemodynamic response function. For the SID/MID, we employed parametric modulation, modeling each trial multiplied by level of reward $(0,1$, or 2$)$ to search for brain regions in which BOLD activity correlated with reward level. Motion estimates were modeled 
as nuisance covariates. Single volume regressors were added to remove volumes exceeding a framewise displacement threshold of $0.9 \mathrm{~mm}$. Time-series statistical analysis was carried out using a general linear model within the FILM module of FSL. Second-level analyses created cross-run averages for each subject's task-level contrast images using a fixed-effects model after normalizing the first-level results to the MNI152 space. The contrast maps thus generated were input into a random-effects model for group analysis, using FLAME1 (FMRIB's Local Analysis of Mixed Effect) with automatic outlier de-weighting.

Main outcomes were tested using a priori defined Region of Interest (ROI) analysis, with small-volume correction (SVC; false discovery rate; FDR). For the EFP, we defined left and right amygdalae ROIs using anatomically defined masks from the Colin27 adult atlas linearly registered to the MNI152 space. For the SID/MID, we defined ROIs using spheres around peak coordinates identified in previous studies. Bilateral nucleus accumbens (NACC) activates reliably during the anticipation of reward, whereas medial prefrontal cortex (mPFC) tracks reward receipt in the MID (Knutson et al, 2003). Given the importance of ventromedial prefrontal cortex (VmPFC) in tracking reward value, we also included an ROI around peak coordinates for vmPFC taken from a meta-analysis of value computation (Clithero and Rangel, 2014). Thus, ROIs for the EFP task consisted of bilateral amygdala, whereas ROIs for the SID and MID comprised bilateral NAcc, MPFC, and vmPFC. In addition to ROI analyses, we present whole brain findings for future hypothesis generation, thresholded at $p<0.001$ with a minimum cluster size of 40 voxels.

\section{RESULTS}


Demographics, Drug Use, and Psychiatric Symptoms

Twenty-two (4 females) COCs and 19 CTRLs (4 females) completed the study. Data from two additional participants were excluded: one male CTRL who disclosed extensive past cocaine and heroin use during debriefing and one male $\mathrm{COC}$ for whom a neuroradiologist review of structural scans indicated significant congenital abnormalities (this subject was referred for clinical follow-up). Participants were well matched on demographic features and psychiatric symptomatology (Table 1).

Drug use data are presented in Table 1. Three CTRLs reported remote cocaine use; two had tried cocaine once, and one reported 3-4 lifetime uses. Groups differed on some indices of drug use: more COCs were current weekly alcohol drinkers and daily cigarette smokers and lifetime weekly cannabis users than CTRLs. Five COCs had tried opioids, one MDMA, one amphetamines, 4 hallucinogens, and 5 PCP, with no past month use of these drugs.

\section{Daily Interpersonal Function}

COCs rated themselves as having more overall interpersonal problems than did $\operatorname{CTRLs}\left(F(1,39)=10.0, p=0.003\right.$, partial $\left.\eta^{2}=0.20\right)$. There was no interaction between IIP subscale and group, suggesting generalized rather than domain-specific difficulties. Similarly, participants' peers reported that COCs had more interpersonal problems overall than CTRLS $\left(F(1,39)=13.9, p=0.001\right.$, partial $\left.\eta^{2}=0.26\right)$, with no interaction between group and IIP subscale (Figure 2). Peers reported that they had known participants for $21.8(\mathrm{SD}=17.1)$ years, with no group difference in average length of relationship. 
Social and Non-Social Threat Processing

One male COC did not undergo scanning; data for threat and reward processing are therefore presented for 21 COCs and 19 CTRLs.

Behaviorally, there was an interaction between threat and the social nature of stimuli $\left(F(1,38)=42.1, p<0.001\right.$, partial $\left.\eta^{2}=0.53\right)$, such that accuracy was lower for threatening relative to non-threatening stimuli in the social category only. There was no main effect of group on EFP accuracy, and no interactions between group, threat, and stimuli type (social vs. non-social). As expected, social threat (angry/fearful faces) compared to neutral stimuli increased bilateral amygdala activation overall; this survived small volume correction for the social threat versus shapes contrast. COCs exhibited higher bilateral amygdala activation to social threat versus shapes relative to CTRLs (Figure 3; Table 2).

Non-social threat increased bilateral amygdala activation relative to non-social nonthreatening stimuli overall, however this did not survive correction. No group differences in amygdala activation in response to non-social threat vs. non-social non-threat survived SVC. Whole brain results for social and non-social threat processing are presented in Table S1. Social and Non-Social Reward Processing

Behaviorally, accuracy and RT in the SID varied linearly with the level of reward (accuracy: $F(1,38)=5.7, p=0.02$, partial $\eta^{2}=0.13 ; \mathrm{RT}: \mathrm{F}(1,38)=5.5, \mathrm{p}=0.025$, partial $\eta^{2}=$ 0.13): accuracy increased and RT decreased with increasing social reward. There was no main effect of group on SID accuracy or RT, and no interactions. Accuracy and RT in the MID also varied linearly with the level of reward in expected directions (accuracy: $F(1,38)=8.2, p$ 
$=0.007$, partial $\eta^{2}=0.18 ; \mathrm{RT}: \mathrm{F}(1,38)=12.7, \mathrm{p}=0.001$, partial $\left.\eta^{2}=0.25\right)$, with no main effects of group or interactions.

As expected, during the SID anticipation phase, level of reward was parametrically tracked in the bilateral NAcc and mPFC across the whole sample. Similarly, BOLD signal in the MPFC, vmPFC and NAcc during MID anticipation was correlated with the level of monetary award available (Figure 4). There were no differences in reward prediction signaling during anticipation between COCs and CTRLs in either SID or MID. During receipt of monetary reward (correct responses only), the level of reward was correlated with BOLD signal in vmPFC across the whole group. In the outcome phase of the SID, vmPFC and mPFC tracked reward magnitude (Figure 5). There were no differences in reward outcome signaling between COCs and CTRLs in the SID or MID. Whole brain results are presented in Table S2.

\section{$\underline{\text { Social Cognition }}$}

There was no main effect of group or interaction of group and emotion on facial emotion recognition accuracy, misclassifications or RTs (all p's $>0.3$ ).

\section{DISCUSSION}

In this study, the first to our knowledge to examine social processing and function in aging drug users, we report that (a) aging cocaine users experience apparent difficulties in day-to-day social function relative to matched non-users, indicated by their own report and that of peers; and (b) cocaine users have heightened amygdala response to social threat 
versus control images compared to non-users. There were no group differences in social or non-social reward processing, or in social cognition, as a function of cocaine use.

Notably, relative to controls, cocaine users had pronounced difficulties in daily social function, whereas performance on laboratory measures was similar with the exception of social threat responding. These findings contrast with previous studies, which reported lowered facial emotion recognition for threatening faces (Ersche et al., 2015; Kemmis et al., 2007; Morgan and Marshall, 2013) as well as blunted social (Preller et al., 2014; Tobler et al., 2016) and non-social (Tobler et al., 2016) reward processing in cocaine users relative to controls. Methodological factors could underlie these differences. For instance, we focused on aging cocaine smokers, whereas previous studies did not. One would expect, however, differences between cocaine users and non-users to be more pronounced in this aging group, where regular cocaine use (weekly or more) was present for at least 15 (and up to 30) years.

An alternative explanation is the breadth of methodological controls for potential confounds employed in this study, including matching on sex, race, age, education, and mood/anxiety symptoms, and controls for recent drug use and current psychopathology. Previous studies have accounted for some, but not all of these factors. It is further possible that the relatively small sample size in this study, which was not powered to detect small effect sizes, may also have contributed to the study outcomes. However, we employed similar or even larger samples to those used in some previous MRI studies that did report substantive differences between cocaine users and non-users in social processing domains

This article is protected by copyright. All rights reserved. 
(e.g. Preller et al., 2014; Tobler et al., 2016). Further research employing larger samples as well as the rigorous methodological controls we employed could more definitively address the reason for discrepancies between the current results and those reported previously

Despite methodological controls, cocaine users did have exaggerated amygdala responses to social threat, consistent with a previous study in cocaine-using males relative to non-using males (Crunelle et al., 2015). In addition to contributing to social difficulties more broadly, hyperactive social threat processing could have implications for psychotherapy, the gold-standard treatment for cocaine users, which relies on social interaction. In some anxiety disorders increased amygdala social threat responding predicts worse treatment outcomes (Bryant et al., 2008).

While the specific etiology of this finding cannot be elucidated based on cross sectional data, several possibilities exist. Heightened sensitivity to social threat may have predated cocaine use, potentially contributing to onset and maintenance of drug use. Supporting this possibility, individuals with conditions associated with cocaine use disorders, such as attention deficit/hyperactivity disorder (Levin et al., 2015), also show exaggerated neural responses to social threat (Posner et al., 2011). Conversely, repeated cocaine or other drug use may contribute to increased social threat responding, either through specific drugrelated neuroadaptations, or via more general neuroplastic alterations associated with the development of addictive behaviors. Future research should address these questions of causality, however, given implications for function and treatment, documenting social processing decrements in drug-using populations is important regardless of etiology.

This article is protected by copyright. All rights reserved. 
As an initial study in this population, there were some limitations. Although we matched samples on demographics, cocaine users had somewhat more co-occurring use of alcohol, cannabis, and cigarettes. This was not unexpected given that polydrug use is common in cocaine users (Vadhan et al., 2014). Second, more males than females entered the study [consistent with prevalence (SAMSHA, 2016)]. Thus, we did not have a large enough sample of females to examine sex differences. Given sex effects on social processing in healthy individuals (Spreckelmeyer et al., 2009), this would be a valuable future direction. Third, while current drug users participated as inpatients, non-drug using controls were offered the option of completing testing as a single outpatient session to ensure adequate recruitment. While there is no reason to conclude this had a systematic effect, it is possible that the inpatient environment preferentially increased social engagement in the cocaine users. Further research should endeavor to test both cocaine users and controls after inpatient stabilization to control for this possibility. Fourth, while candidates across groups were excluded for current mood, anxiety and eating disorders and lifetime mania and psychosis, we did not assess personality disorders, which are strongly implicated in social processing (e.g. Herpertz and Bertsch, 2014). The close similarity in current mood and anxiety symptoms, as well as the absence of prominent differences between the groups on most social processing measures are somewhat reassuring in this regard. However, we cannot conclusively rule out possible effects of undetected personality disorders on the study outcomes. Finally, inclusion of younger cocaine users and controls, allowing investigation of

This article is protected by copyright. All rights reserved. 
interactive effects of age and cocaine use on social function, was beyond the scope of this study.

These data, the first to examine social processing and function in aging drug users, indicate that older cocaine users have marked difficulties in daily social function but largely intact social cognition and motivational processing when tested under well-controlled conditions and compared to appropriately-matched controls. Findings underline the importance of careful experimental control in studies of this type, and support interpersonal function as a valuable domain for future research on aging drug users.

This article is protected by copyright. All rights reserved. 
Funding and Disclosures: This work was supported by the National Institute on Drug Abuse (DA030540 and DA034877). The authors have no conflicts of interest to declare.

Acknowledgements: The authors would like to thank the volunteers for participating and Jolie Gorchov and Amy Curcio for assistance in data collection.

This article is protected by copyright. All rights reserved. 
Table 1. Demographics, Psychiatric Symptoms, and Drug Use

\begin{tabular}{|c|c|c|c|}
\hline & $\begin{array}{c}\text { Cocaine Users } \\
N=22\end{array}$ & $\begin{array}{c}\text { Controls } \\
N=19\end{array}$ & \\
\hline & $N(\%)$ & $N(\%)$ & $X^{2}(d f)$ \\
\hline Sex, Female & $4(18)$ & $4(21)$ & $0.1(1)$ \\
\hline Race, AA & $22(100)$ & $18(95)$ & $1.2(1)$ \\
\hline \multirow[t]{2}{*}{ Ethnicity, non-Hispanic } & $22(100)$ & $19(100)$ & N/A \\
\hline & Mean (S.D.) & Mean (S.D.) & $t(d f)$ \\
\hline Age & $52.9(2.5)$ & $52.7(2.6)$ & $0.2(39)$ \\
\hline Education, years & $13.3(1.7)$ & $14.1(2.0)$ & $1.5(39)$ \\
\hline Depression Sx: BDI & $4.5(5.5)$ & $3.0(4.1)$ & $1.0(39)$ \\
\hline Anxiety Sx: STICSA & $24.5(4.1)$ & $23.4(2.9)$ & $1.0(39)$ \\
\hline Trauma Exposure: TAA & $1.2(1.3)$ & $2.4(2.1)$ & $2.1(28.1)^{\#}$ \\
\hline Positive Affect: PANAS & $35.2(9.5)$ & $39.3(7.6)^{a}$ & $1.5(38)$ \\
\hline Negative Affect: PANAS & $11.9(2.7)$ & $10.6(1.1)^{b}$ & $2.0(29.5)^{\#}$ \\
\hline Past Month Cocaine Use (days/week) & $3.9(1.4)$ & - & - \\
\hline $\begin{array}{l}\text { Past Month Weekly Cocaine Cost } \\
\text { (USD) }\end{array}$ & $\begin{array}{l}\$ 258.64 \\
(\$ 225.66)\end{array}$ & - & - \\
\hline Heaviest Cocaine Use (days/week) & $5.9(1.6)$ & - & - \\
\hline $\begin{array}{l}\text { Heaviest Weekly Cocaine Cost } \\
\text { (USD) }\end{array}$ & $\begin{array}{l}\$ 695.46 \\
(\$ 624.32)\end{array}$ & - & - \\
\hline \multirow[t]{2}{*}{$\begin{array}{l}\text { Years Regular Cocaine Use } \\
\text { ( } \geq \text { weekly) }\end{array}$} & $21.7(4.4)$ & - & - \\
\hline & $N(\%)$ & $N(\%)$ & $X^{2}(d f)$ \\
\hline Past Month Weekly Alcohol Use & $17(77)$ & $4(21)$ & $12.9(1)^{\star}$ \\
\hline
\end{tabular}

This article is protected by copyright. All rights reserved. 


\begin{tabular}{|c|c|c|c|}
\hline Lifetime Weekly Alcohol Use & $17(77)$ & $10(53)$ & $2.8(1)$ \\
\hline Past Month Weekly Cannabis Use & $3(14)$ & $0(0)$ & $2.8(1)$ \\
\hline Lifetime Weekly Cannabis Use & $16(73)$ & $6(32)$ & $6.9(1)^{\star}$ \\
\hline Past Month Daily Cigarette Use & $15(68)$ & $5(26)$ & $7.2(1)^{\star}$ \\
\hline & Mean (S.D.) & Mean (S.D.) & $t(d f)$ \\
\hline Past Month Alcohol Use (days/week) & $2.8(1.4)$ & $2.6(0.9)$ & $0.3(19)$ \\
\hline $\begin{array}{l}\text { Highest Frequency Alcohol Use } \\
\text { (days/week) }^{c}\end{array}$ & $4.8(2.4)$ & $4.2(2.7)$ & $0.7(25)$ \\
\hline $\begin{array}{l}\text { Past Month Cannabis Use } \\
\text { (days/week) }^{c}\end{array}$ & $3.5(3.1)$ & $\mathrm{N} / \mathrm{A}$ & N/A \\
\hline $\begin{array}{l}\text { Highest Frequency Cannabis Use } \\
\text { (days/week) }^{c}\end{array}$ & $6.4(1.5)$ & $3.8(2.5)$ & $2.4(6.5)^{\#}$ \\
\hline $\begin{array}{l}\text { Past Month Cigarette Smoking } \\
\text { (cigarettes/day) }^{d}\end{array}$ & $7.8(5.5)$ & $7.2(3.0)$ & $0.2(18)$ \\
\hline \multicolumn{4}{|c|}{$\begin{array}{l}\text { BDI = Beck Depression Inventory; STICSA = State Trait Inventory of Cognitive and Somatic } \\
\text { Anxiety; TAA = Trauma Assessment for Adults; PANAS = Positive and Negative Affect } \\
\text { Schedule; N/A = not applicable (all participants were non-Hispanic); Sx = symptoms. } \\
\# p=0.05 \\
\text { * } p<0.05 \\
\text { a } n=18 \text { due to missing data. } \\
{ }^{b} n=17 \text { due to missing data. } \\
{ }^{c} \text { Data from those who reported regular (at least weekly) use of alcohol or cannabis within the } \\
\text { specified time frame were included; } \\
\text { d } \text { Only data from those reporting current daily cigarette smoking were included. }\end{array}$} \\
\hline
\end{tabular}

This article is protected by copyright. All rights reserved. 
Table 2. Group differences in Threat Processing: Region of Interest findings

Social Threat $>$ Shape Contrast, Cocaine $>$ Control

\begin{tabular}{l|c|c|c|c}
\hline \multicolumn{1}{c|}{ Region } & Cluster Size & $\begin{array}{c}\text { Center } \\
\text { Coordinates } \\
(\text { MNI) }\end{array}$ & Max. p value & $\begin{array}{c}\text { Max. } \\
\text { Coordinates } \\
\text { (MNI) }\end{array}$ \\
\hline $\begin{array}{l}\text { Amygdala, } \\
\text { right }\end{array}$ & 231 voxels & $26,-2,-20$ & 0.0004 & $28,-6,-18$ \\
Amygdala, left & 92 voxels & $-19,-4,-19$ & 0.001 & $-18,-8,-16$ \\
\hline
\end{tabular}

This article is protected by copyright. All rights reserved. 


\section{Figure Legends}

Figure 1. Schematic of Social Incentive Delay (SID) task, adapted from Spreckelmeyer et al (2009).

Figure 2. Daily interpersonal problems in cocaine users and controls measured via self- and peer-report. Data are means; error bars are S.E.M. Asterisks indicate statistically significant differences between groups $(p<0.005)$.

Figure 3. Amygdala response to social threat versus shape stimuli (A) across the whole group (upper panel) and (B) in cocaine relative to versus controls (bottom panel). Analyses employed small volume correction across a priori defined amygdalae ROls.

Figure 4. Brain regions tracking reward prediction signals (i.e. BOLD signal correlated with reward magnitude during anticipation) during $(A)$ the Monetary Incentive Delay task (upper panel) and (B) the Social Incentive Delay task (bottom panel) across the whole group. Analyses employed small volume correction across a priori defined ROIs (vmPFC = ventromedial PreFrontal Cortex; mPFC = medial PreFrontal Cortex; NAcc = Nucleus Accumbens). There were no differences in reward prediction encoding between the COC and CTRL groups in either the SID or the MID.

Figure 5. Brain regions tracking reward receipt signals (i.e. BOLD signal correlated with reward magnitude during outcome) during (A) the Monetary Incentive Delay task (upper panel) and (B) the Social Incentive Delay task (bottom panel) across the whole group. Analyses employed small volume correction across a priori defined ROIs (vmPFC = 
ventromedial PreFrontal Cortex; $\mathrm{mPFC}=$ medial PreFrontal Cortex $; \mathrm{NAcc}=$ Nucleus

Accumbens). There were no differences in reward receipt encoding between the $\mathrm{COC}$ and CTRL groups in either the SID or the MID.

\section{References}

Afful S, Strickland J, Cottler LB, Beirut L (2010) Exposure to trauma: A comparison of cocaine-dependent cases and a community-matched sample. Drug and Alcohol Dependence 112:46-53.

APA (1994) DSM-IV: Diagnostic and Statistical Manual of Mental Disorders. American Psychiatric Association: MD, US.

Beck AT, Steer RA (1993) Manual for the Beck Depression Inventory. The Psychological Corporation: San Antonio, US.

Bedi G, Shiffrin L, Vadhan NP, Nunes EV, Foltin RW, Bisaga A (2016) Effects of levodopacarbidopa-entacapone and smoked cocaine on facial affect recognition in cocaine smokers. J Psychopharmacol 30:370-377.

Bryant RA, Felmingham K, Kemp A, Das P, Hughes G, Peduto A, Williams L (2008) Amygdala and ventral anterior cingulate activation predicts treatment response to cognitive behaviour therapy for post-traumatic stress disorder. Psychol Med 38:555-561.

Churchill NW, Strother SC (2013) PHYCAA+: an optimized, adaptive procedure for measuring and controlling physiological noise in BOLD fMRI. Neuroimage 82:306-325. Cornish JW, O'Brien CP (1996) Crack cocaine abuse: an epidemic with many public health consequences. Annual review of public health 17:259-273.

Crunelle CL, Kaag AM, van den Munkhof HE, Reneman L, Homberg JR, Sabbe B, van den Brink W, van Wingen G (2015) Dysfunctional amygdala activation and connectivity with the prefrontal cortex in current cocaine users. Hum Brain Mapp 36:4222-4230.

Cunha PJ, Bechara A, de Andrade AG, Nicastri S (2011) Decision-making deficits linked to real-life social dysfunction in crack cocaine-dependent individuals. The American journal on addictions / American Academy of Psychiatrists in Alcoholism and Addictions 20:78-86. Cusack KJ, Frueh BC, Brady KT (2004) Trauma history screening in a community mental health center. Psychiatr Serv 55:157-162.

Dowling G, Weiss S, Condon T (2008) Drugs of abuse and the aging brain. Neuropsychopharmacology 33:209-218.

Eiden R, Foote A, Schuetze P (2007) Maternal cocaine use and caregiving status: Group differences in caregiver and infant risk variables. Addictive Behaviors 32:465-476. 
Ersche KD, Hagan CC, Smith DG, Jones PS, Calder AJ, Williams GB (2015) In the face of threat: neural and endocrine correlates of impaired facial emotion recognition in cocaine dependence. Transl Psychiatry 5:e570.

Eyrich-Garg K, Cacciola J, Carise D, Lynch K, McLellan A (2008) Individual characteristics of the literally homeless, marginally housed, and improverished in a US substance abuse treatment-seeking sample. . Social Psychiatry and Psychiatric Epidemiology 43:831-842. First M, Spitzer R, Gibbon M, Williams J (2002) Structured Clinical Interview for DSM-IV-TR Axis I Disorders, Research Version, Patient Edition. . Biometrics Research: New York. Fuller-Iglesias HR, Rajbhandari S (2016) Development of a Multidimensional Scale of Social Integration in Later Life. Research on aging 38:3-25.

Gfroerer J, Brodsky M (1993) Frequent cocaine users and their use of treatment. American Journal of Public Health 83:1149-1154.

Glover GH, Law CS (2001) Spiral-in/out BOLD fMRI for increased SNR and reduced susceptibility artifacts. Magnetic resonance in medicine : official journal of the Society of Magnetic Resonance in Medicine / Society of Magnetic Resonance in Medicine 46:515-522. Han B, Gfroerer JC, Colliver JD, Penne MA (2009) Substance use disorder among older adults in the United States in 2020. Addiction 104:88-96.

Hariri A, Tessitore A, Mattay V, Fera F, Weinberger D (2002) The amygdala response to emotional stimuli: A comparison of faces and scenes. Neurolmage 17:317-323.

Herpertz SC, Bertsch K (2014) The social-cognitive basis of personality disorders. Curr Opin Psychiatry 27:73-77.

Horowitz L, Alden L, Wiggins J, Pincus A (2000) Inventory of Interpersonal Problems Manual and Sampler Set. Mind Garden, Inc: Menlo Park, CA.

Hulka LM, Preller KH, Vonmoos M, Broicher SD, Quednow BB (2013) Cocaine users manifest impaired prosodic and cross-modal emotion processing. Frontiers in psychiatry 4:98.

Kalapatapu RK, Vadhan NP, Rubin E, Bedi G, Cheng WY, Sullivan MA, Foltin RW (2011) A pilot study of neurocognitive function in older and younger cocaine abusers and controls. The American journal on addictions / American Academy of Psychiatrists in Alcoholism and Addictions 20:228-239.

Kemmis L, Hall J, Kingston R, Morgan M (2007) Impaired fear recognition in regular recreational cocaine users. Psychopharmacology 194:151-159.

Knutson B, Westdorp A, Kaiser E, Hommer D (2000) fMRI visualization of brain activity during a monetary incentive delay task. Neurolmage 12:20-27.

Kornreich C, Philippot P, Foisy M-L, Blairy S, Raynaud E, Dan Bea (2002) Impaired emotional face expression recognition is associated with interpersonal problems in alcoholism. Alcohol and Alcoholism 37:394-400.

Kouimtsidis C, Padhi A (2007) A case of late-onset dependence on cocaine and crack. Addiction 102:666-667. 
Kuhns J, Wilson D, Maguire E, Ainsworth S, Clodfelter T (2009) A meta-analysis of marijuana, cocaine and opiate toxicology study findings among homicide victims. Addiction 104:1122-1131.

Kuypers KP, Steenbergen L, Theunissen EL, Toennes SW, Ramaekers JG (2015) Emotion recognition during cocaine intoxication. European neuropsychopharmacology : the journal of the European College of Neuropsychopharmacology 25:1914-1921.

Lang PJ, Bradley MM, Cuthbert BN (1997) International Affective Picture System (IAPS):

Technical Manual and Affective Ratings Center for the Study of Emotion and Attention, University of Florida: Gainesville, FL.

Levin FR, Mariani JJ, Specker S, Mooney M, Mahony A, Brooks DJ, Babb D, Bai Y, Eberly LE, Nunes EV, Grabowski J (2015) Extended-Release Mixed Amphetamine Salts vs Placebo for Comorbid Adult Attention-Deficit/Hyperactivity Disorder and Cocaine Use Disorder: A Randomized Clinical Trial. JAMA psychiatry 72:593-602.

Moore T, Stuart G, Meehan J, Rhatigan D, Hellmuth J, Keen S (2008) Drug abuse and aggression between intimate partners: A meta-analytic review. Clinical Psychology Review 28:247-274.

Morgan MJ, Marshall JP (2013) Deficient fear recognition in regular cocaine users is not attributable to elevated impulsivity or conduct disorder prior to cocaine use. J

Psychopharmacol 27:526-532.

Posner J, Nagel BJ, Maia TV, Mechling A, Oh M, Wang Z, Peterson BS (2011) Abnormal amygdalar activation and connectivity in adolescents with attention-deficit/hyperactivity disorder. Journal of the American Academy of Child and Adolescent Psychiatry 50:828-837 e823.

Preller KH, Herdener M, Schilbach L, Stampfli P, Hulka LM, Vonmoos M, Ingold N, Vogeley K, Tobler PN, Seifritz E, Quednow BB (2014) Functional changes of the reward system underlie blunted response to social gaze in cocaine users. Proceedings of the National Academy of Sciences of the United States of America 111:2842-2847.

Rao R, Roche A (2017) Substance misuse in older people. BMJ 358:j3885.

Ratcliff R (1993) Methods for dealing with reaction time outliers. Psychol Bull 114:510-532. Rosen D, Engel RJ, Hunsaker AE, Engel Y, Detlefsen EG, Reynolds CF, 3rd (2013) Just say know: an examination of substance use disorders among older adults in gerontological and substance abuse journals. Social work in public health 28:377-387.

SAMSHA (2016) Results from the 2015 National Survey on Drug Use and Health: Detailed Tables. Substance Abuse and Mental Health Services Administration, Department of Health and Human Services: Rockville, MD.

Spreckelmeyer K, Krach S, Kohls G, Rademacher L, A I, al. KKe (2009) Anticipation of monetary and social reward differentially activates mesolimbic brain structures in men and women. Social Cognitive and Affective Neuroscience 4:158-165.

Tobler PN, Preller KH, Campbell-Meiklejohn DK, Kirschner M, Kraehenmann R, Stampfli P, Herdener M, Seifritz E, Quednow BB (2016) Shared neural basis of social and non-social reward deficits in chronic cocaine users. Soc Cogn Affect Neurosci 11:1017-1025. 
Tottenham N, Tanaka JW, Leon AC, McCarry T, Nurse M, Hare TA, Marcus DJ, Westerlund A, Casey BJ, Nelson C (2009) The NimStim set of facial expressions: judgments from untrained research participants. Psychiatry Res 168:242-249.

Vadhan NP, Myers CE, Benedict E, Rubin E, Foltin RW, Gluck MA (2014) A decrement in probabilistic category learning in cocaine users after controlling for marijuana and alcohol use. Exp Clin Psychopharmacol 22:65-74.

Van Dam NT, Gros DF, Earleywine M, Antony MM (2013) Establishing a trait anxiety threshold that signals likelihood of anxiety disorders. Anxiety, stress, and coping 26:70-86. Volkow ND, Baler RD, Goldstein RZ (2011) Addiction: pulling at the neural threads of social behaviors. Neuron 69:599-602.

Watson D, Clark LA, Tellegen A (1988) Development and validation of brief measures of positive and negative affect: The PANAS scales. Journal of personality and social psychology 54:1063-1070.

Yarnell SC (2015) Cocaine Abuse in Later Life: A Case Series and Review of the Literature. The primary care companion for CNS disorders 17.

This article is protected by copyright. All rights reserved. 


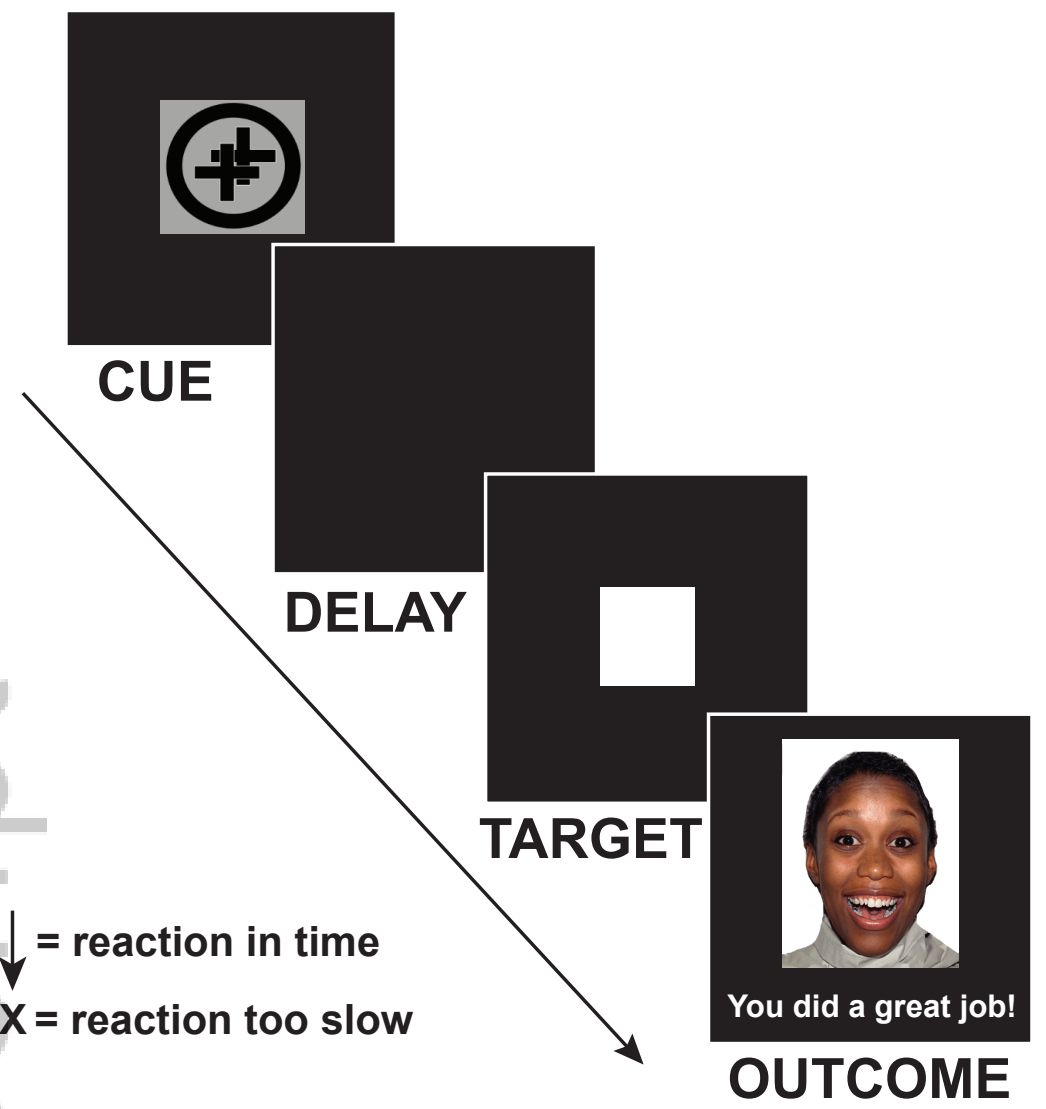

\section{No Reward}

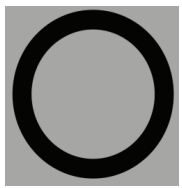

$\downarrow x$

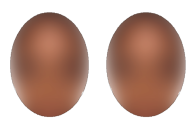

Low Reward
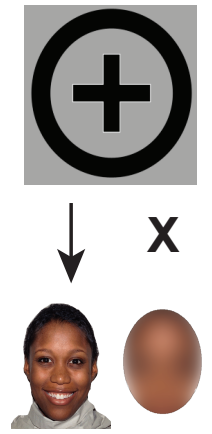

High Reward
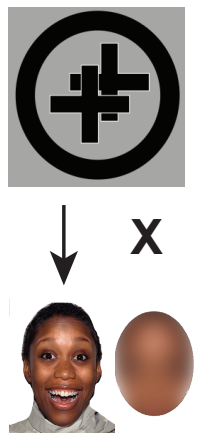


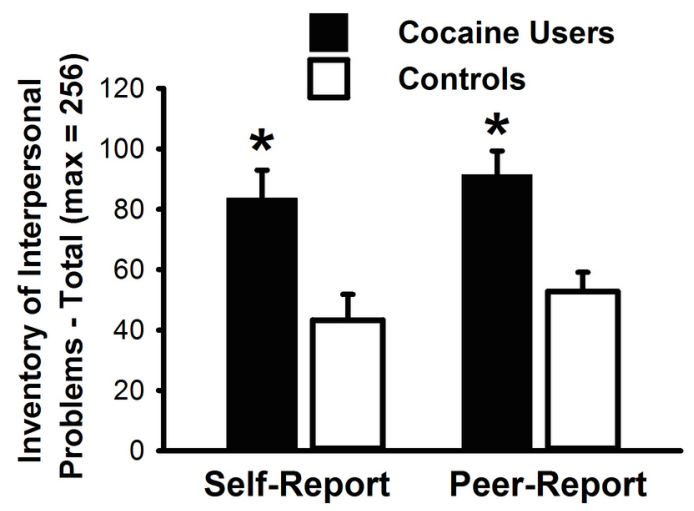

ADB_12669_f2.JPG

This article is protected by copyright. All rights reserved. 
$\mathbf{A}$
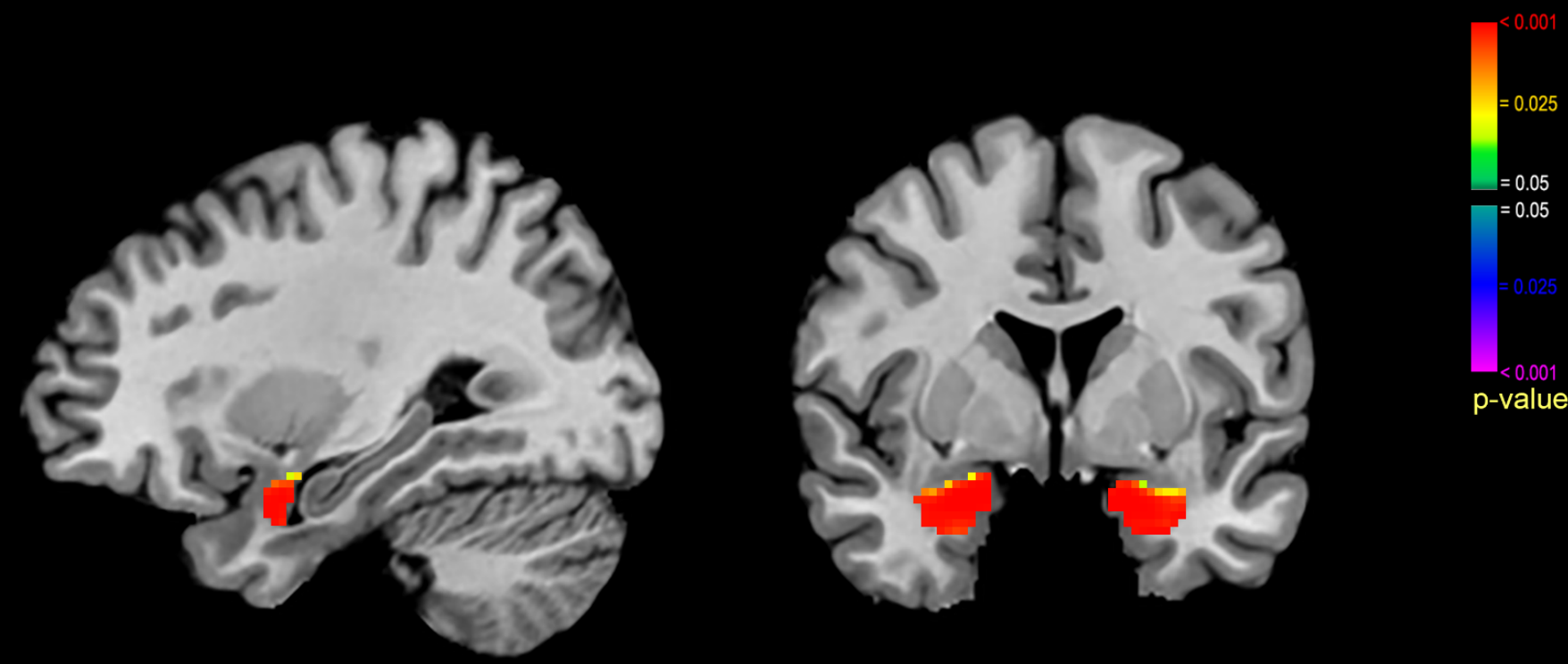

\section{$X=28$}

\section{$\mathbf{Y}=\mathbf{0}$}

B
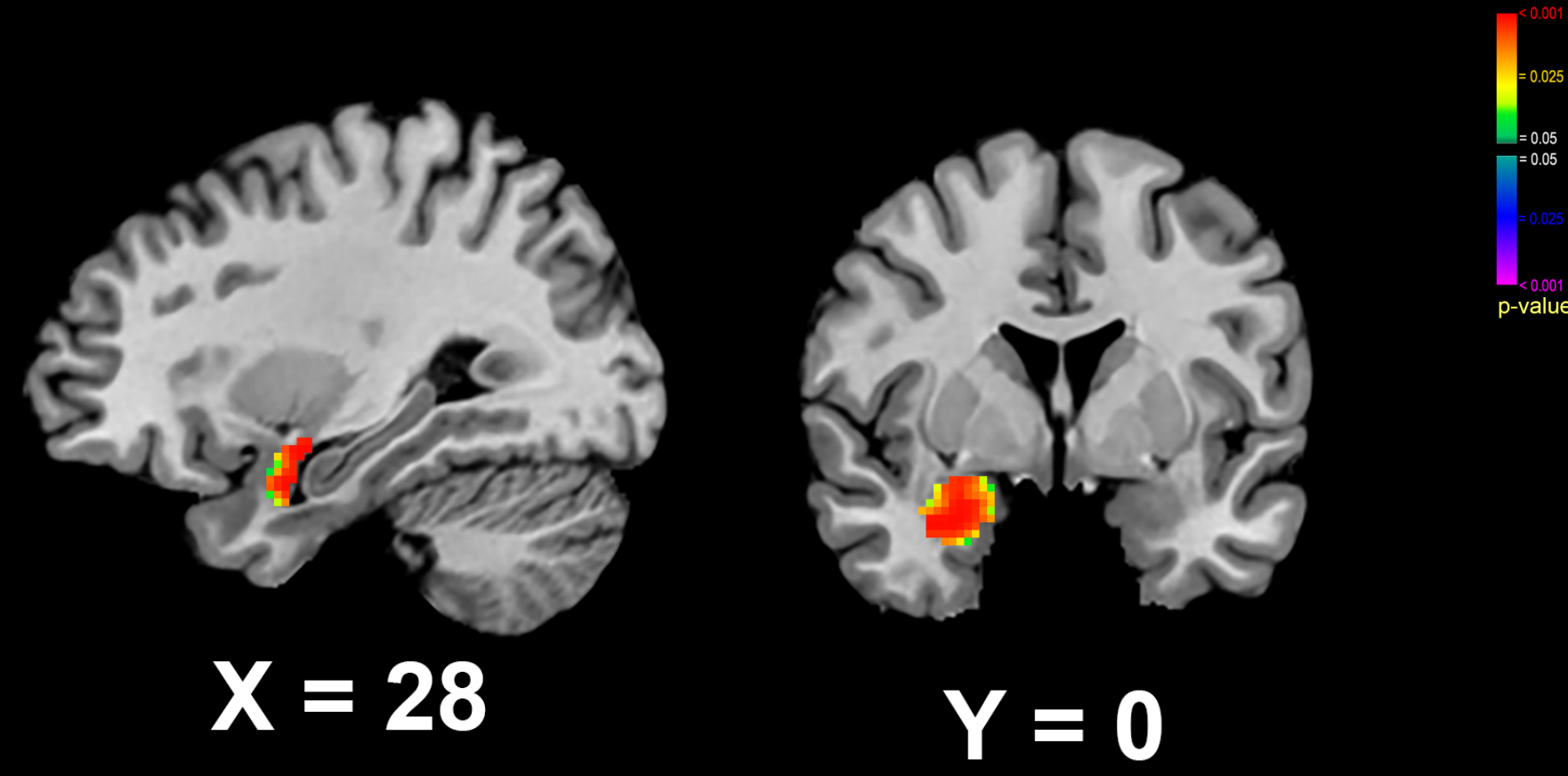
A.
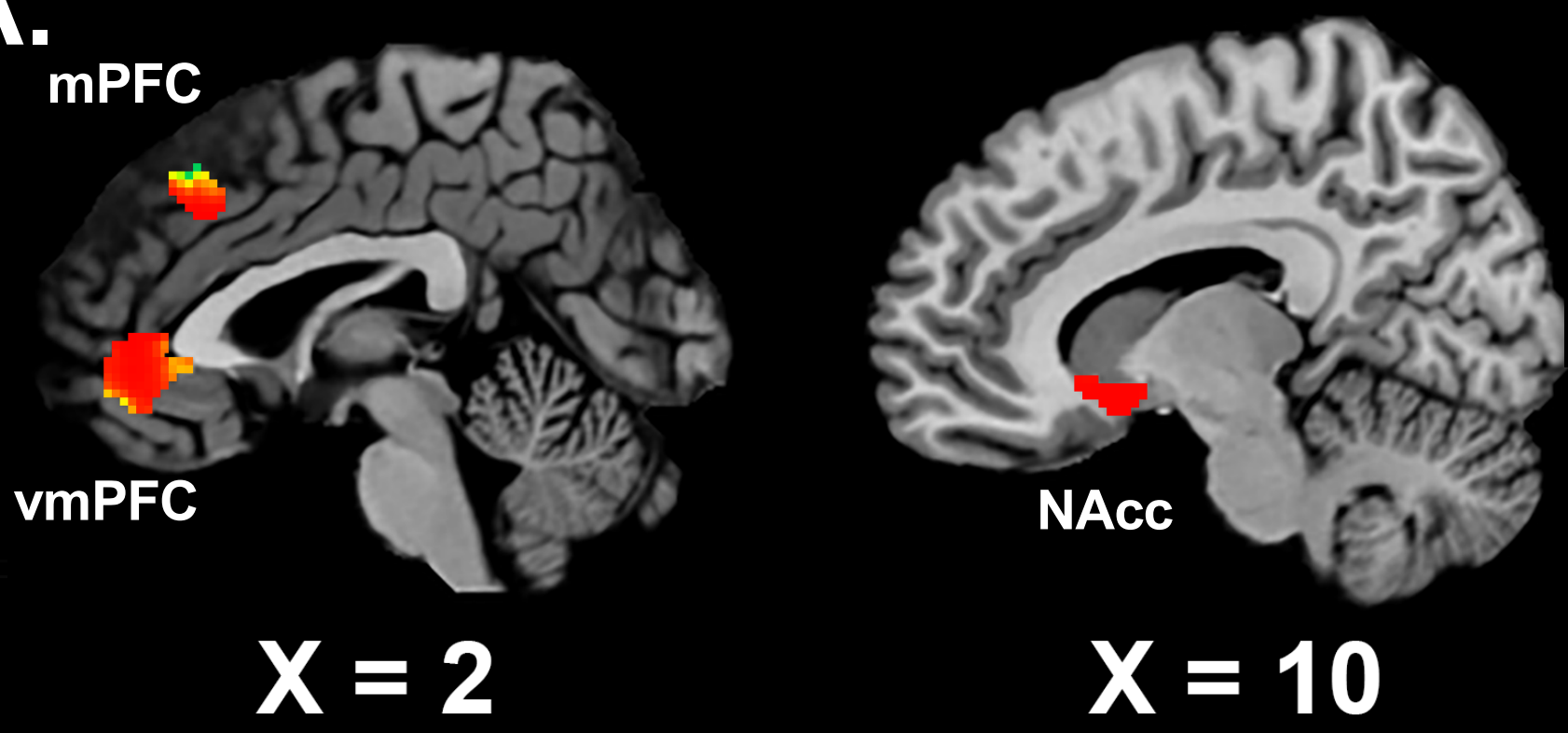

B.
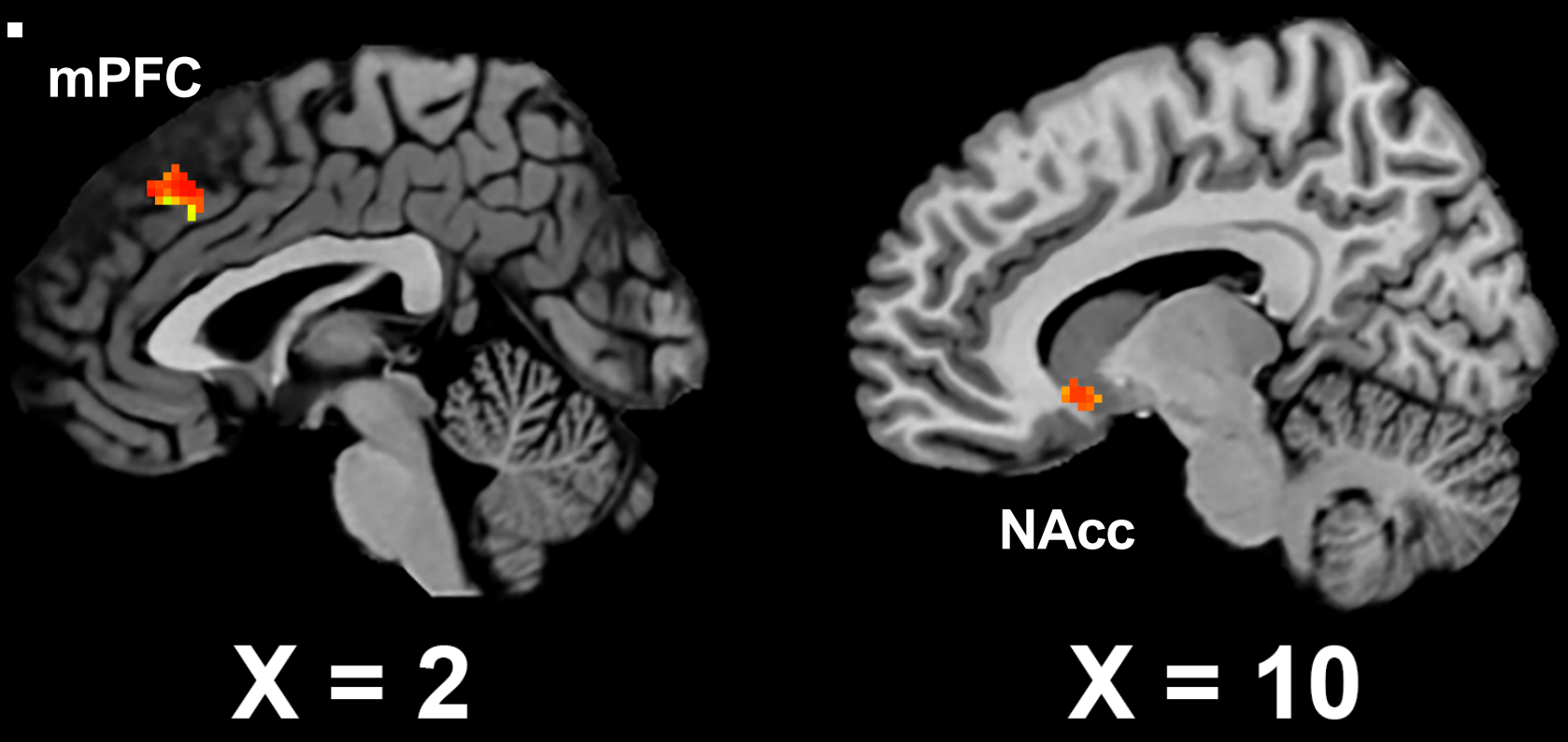
A.
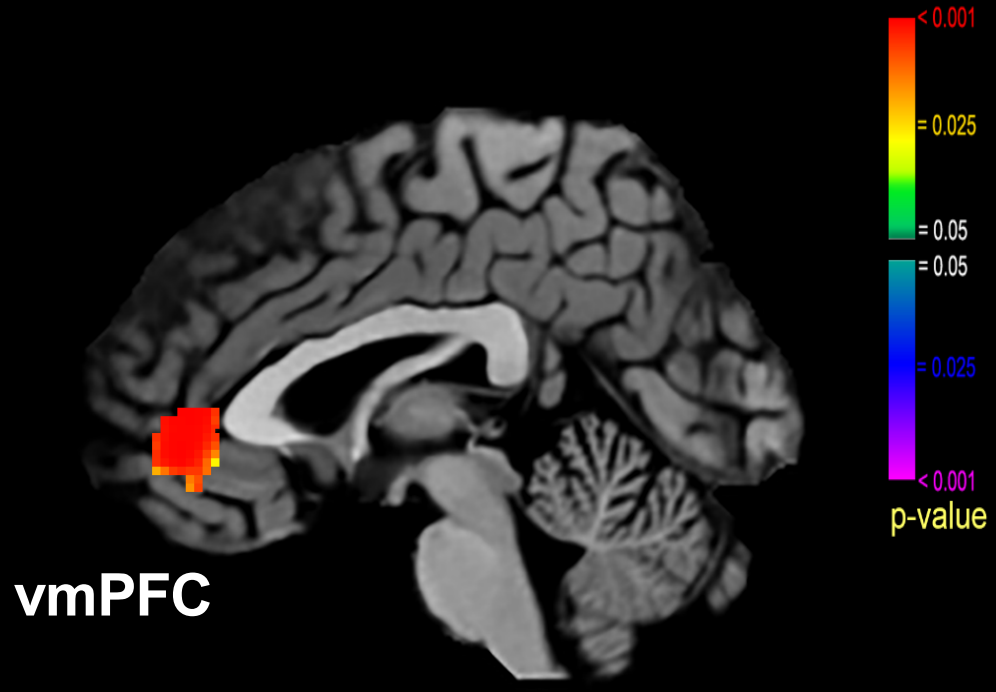

$X=2$

B.

MPFC

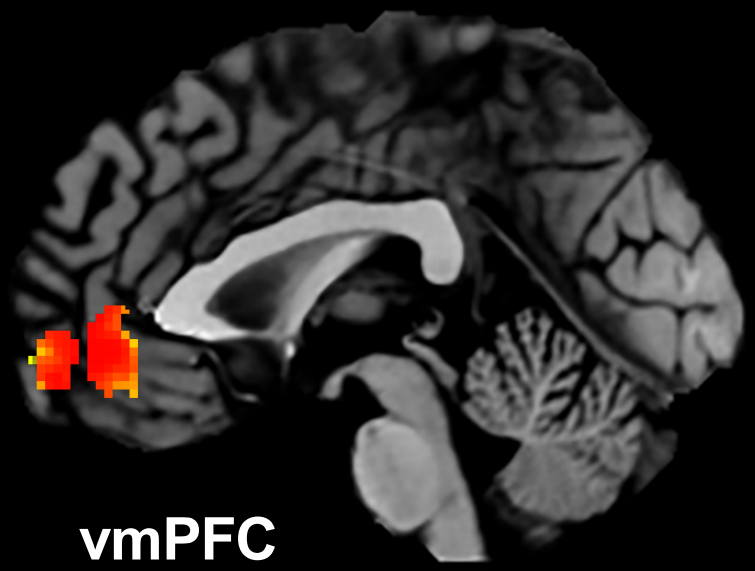

$x=0$ 


\section{University Library}

\section{- M M N E R VA A gateway to Melbourne's research publications}

Minerva Access is the Institutional Repository of The University of Melbourne

Author/s:

Bedi, G;Hao, X;Van Dam, NT;Cooper, ZD;Rubin, E;Vadhan, NP;Marino, L;Haney, M

Title:

Social motivational processing and interpersonal function in aging cocaine smokers

Date:

2019-09-01

Citation:

Bedi, G., Hao, X., Van Dam, N. T., Cooper, Z. D., Rubin, E., Vadhan, N. P., Marino, L. \& Haney, M. (2019). Social motivational processing and interpersonal function in aging cocaine smokers. ADDICTION BIOLOGY, 24 (5), pp.1044-1055. https://doi.org/10.1111/ adb.12669.

Persistent Link:

http://hdl.handle.net/11343/284622 